\title{
ANÁLISE ESPACIAL E EPIDEMIOLÓGICA DE HEPATITES B E C E ÍNDICE DE DESENVOLVIMENTO HUMANO MUNICIPAL, NO ESTADO DO PARÁ
}

\section{SPATIAL AND EPIDEMIOLOGICAL ANALYSIS OF HEPATITIS B AND C AND MUNICIPAL HUMAN DEVELOPMENT INDEX IN THE STATE OF PARA}

\begin{abstract}
Nelson Veiga Gonçalves
Universidade Federal Rural da Amazônia, Instituto Ciberespacial, Belém, Pará, Brasil

Universidade do Estado do Pará, Centro de Ciências Biológicas e da Saúde

Departamento de Saúde Comunitária, Laboratório de Epidemiologia e Geoprocessamento, Belém, Pará, Brasil nelsoncg2009@gmail.com

Dayseanne Costa Vieira

Universidade do Estado do Pará, Centro de Ciências Biológicas e da Saúde Departamento de Saúde Comunitária, Laboratório de Epidemiologia e Geoprocessamento, Belém, Pará, Brasil epigeo@gmail.com
\end{abstract}

Claudia do Socorro Carvalho Miranda

Universidade do Estado do Pará, Centro de Ciências Biológicas e da Saúde Departamento de Saúde Comunitária, Laboratório de Epidemiologia e Geoprocessamento, Belém, Pará, Brasil cllaumiranda@gmail.com

Vera Regina da Cunha Menezes Palácios Universidade do Estado do Pará, Centro de Ciências Biológicas e da Saúde Departamento de Saúde Comunitária, Laboratório de Epidemiologia e Geoprocessamento, Belém, Pará, Brasil verareginapalacios@gmail.com

Simone Beverly Nascimento da Costa Universidade do Estado do Pará, Centro de Ciências Biológicas e da Saúde Departamento de Saúde Comunitária, Belém, Pará, Brasil beverlycosta@hotmail.com

Juan Andrade Guedes

Universidade do Estado do Pará, Centro de Ciências Biológicas e da Saúde Departamento de Saúde Comunitária, Laboratório de Epidemiologia e Geoprocessamento, Belém, Pará, Brasil juan andrade guedes@hotmail.com

Bruno de Oliveira Santos

Universidade do Estado do Pará, Centro de Ciências Biológicas e da Saúde Departamento de Saúde Comunitária, Laboratório de Epidemiologia e Geoprocessamento, Belém, Pará, Brasil broliveirasantos@hotmail.com

Rodrigo Junior Farias da Costa

Universidade do Estado do Pará, Centro de Ciências Biológicas e da Saúde Departamento de Saúde Comunitária, Laboratório de Epidemiologia e Geoprocessamento, Belém, Pará, Brasil rodrigofjunior@hotmail.com

Sheila Cristina Martins e Silva

Universidade do Estado do Pará, Centro de Ciências Biológicas e da Saúde Departamento de Saúde Comunitária, Laboratório de Epidemiologia e Geoprocessamento, Belém, Pará, Brasil nutricsheilamartins@gmail.com

Rafael Aleixo Coelho de Oliveira Graduando em Engenharia Ambiental. Universidade do Estado do Pará Centro de Ciências Biológicas e da Saúde Departamento de Saúde Comunitária, Laboratório de Epidemiologia e Geoprocessamento, Belém, Pará, Brasil aleixoralaelll@gmail.com

Recebido em: 30/11/2018

Aceito para publicação em: 02/07/2019 
Análise espacial e epidemiológica de hepatites $\mathrm{B}$ e $\mathrm{C}$ e índice de desenvolvimento humano municipal, no estado do Pará
Nelson Veiga Gonçalves

Dayseanne Costa Vieira

Claudia do Socorro Carvalho Miranda

Vera Regina da Cunha Menezes Palácios

Simone Beverly Nascimento da Costa

Juan Andrade Guedes

Bruno de Oliveira Santos

Rodrigo Junior Farias da Costa

Sheila Cristina Martins e Silva

Rafael Aleixo Coelho de Oliveira

\begin{abstract}
RESUMO
Considerando as hepatites B e C um grave problema de saúde pública no Brasil, este estudo objetivou analisar a distribuição espacial e socioeconômica destas doenças, nos municípios atendidos por três Centros Regionais de Saúde (CRS), da Secretaria de Saúde Pública do Estado do Pará, de 2010 a 2014. Foi realizado um estudo descritivo e transversal com dados do Ministério da Saúde e do Instituto Brasileiro de Geografia e Estatística. As análises epidemiológicas mostraram que o 1ํㅡㄴ notificou maior número de casos, sobretudo em 2012 e 2014. O perfil dos indivíduos mais acometidos foi sexo masculino $(58,9 \%)$, pardos $(65,1 \%)$, adultos $(65,6 \%)$, baixa escolaridade $(27,3 \%)$ e residentes em zona urbana (93,0\%). Os fatores de riscos mais significativos nos três CRS foram as doenças sexualmente transmissíveis e uso de medicamentos injetáveis. A análise espacial entre os parâmetros do IDHM e da taxa de incidência mostrou uma autocorrelação inversa entre essas duas variáveis. Foi observado que áreas com IDHM médio e baixo apresentaram altas taxas de incidência. As análises geradas foram eficazes para construir um cenário epidemiológico contextualizado das hepatites $\mathrm{B}$ e C. Ressaltamos a necessidade de expansão de programas de controle destas doenças nas áreas estudadas.
\end{abstract}

Palavras-Chave: Hepatite B. Hepatite C. Epidemiologia. Análise Espacial.

\begin{abstract}
Considering hepatitis $\mathrm{B}$ and $\mathrm{C}$ a serious public health problem in Brazil, this study aimed to analyze the spatial and socioeconomic distribution of those diseases, in the municipalities attended by three Regional Health Centers $(\mathrm{RHC})$ of the Public Health Secretary of the state of Para, from 2010 to 2014. A descriptive and cross-sectional study was carried out with data from the Health Ministry and from the Brazilian Institute of Geography and Statistics. The epidemiological analyzes showed that the 1st CRS reported the highest number of cases, especially in 2012 and 2014 . The most affected individuals profile was male (58,9\%), brown $(65,1 \%)$, adults $(65,6 \%)$, low schooling $(27,3 \%)$ and urban area resident $(93,0 \%)$. The most significant risk factors in the three RHC were sexually transmitted diseases and use of injectable drugs. The spatial analyzes between the parameters of MHDI and of the incidence rate showed an inverse autocorrelation between these two variables. It was observed that areas with medium and low MHDI presented high incidence rates. The analyzes generated were effective to construct a contextualized epidemiological scenario of the hepatitis $B$ and C. We emphasize the need of expansion of those diseases control programs in the studied areas.
\end{abstract}

Keywords: Hepatitis B. Hepatitis C. Epidemiology. Spatial Analysis.

\title{
INTRODUÇÃO
}

As hepatites B e C são doenças infecciosas, causadas pelos vírus das hepatites B (HBV) e C (HCV), que constituem um grande problema de saúde pública no mundo, sendo hoje as causas mais comuns de indicação de transplante hepático. As transmissões do HBV e do HCV ocorrem de forma semelhante por via percutânea e por transmissão horizontal ou vertical (BENSABATH; LEÃO, 2003; BRASIL, 2017; LOPES; SCHINONI, 2011). A Organização Mundial de Saúde (OMS) estimou que 257 milhões de pessoas estavam infectadas por HBV e 71 milhões por HCV em 2015 (OMS, 2017). De 2000 a 2015, foram identificados, no Brasil, pelo Sistema de Informação de Mortalidade (SIM), 61.297 óbitos associados às hepatites virais. Desses, $21,6 \%$ foram associados à hepatite $\mathrm{B}$ e $75,6 \%$ à hepatite C (BRASIL, 2017).

Segundo o Boletim Epidemiológico das hepatites virais (Brasil, 2017), no período de 1999 a 2011, foram notificados no Sistema de Informação de Agravos de Notificação (SINAN) 15.571 casos de hepatite B na Região Norte, o que corresponde a 13,1\% do total de casos no Brasil. Neste mesmo 
Análise espacial e epidemiológica de hepatites B e $\mathrm{C}$ e índice de desenvolvimento humano municipal, no estado do Pará
Nelson Veiga Gonçalves

Dayseanne Costa Vieira

Claudia do Socorro Carvalho Miranda

Vera Regina da Cunha Menezes Palácios

Simone Beverly Nascimento da Costa

Juan Andrade Guedes

Bruno de Oliveira Santos

Rodrigo Junior Farias da Costa

Sheila Cristina Martins e Silva

Rafael Aleixo Coelho de Oliveira

sistema, no período de 1999 a 2010, foram notificados 1.644 casos confirmados de hepatite $C$, nesta mesma região, que representa $2 \%$ do total de casos notificados no país.

O Brasil, ao longo de sua história, tem apresentado grandes diferenças em seus indicadores socioeconômicos, que têm influenciado a distribuição geográfica de várias doenças infecciosas e parasitárias. Desta forma, o estudo da relação entre esses indicadores tem se constituído de um grande desafio para a saúde coletiva, sobretudo, quando consideramos as escalas locais dos territórios nacionais.

Diversos estudos têm procurado analisar a relação entre o Índice de Desenvolvimento Humano (IDH) e a prevalência destas doenças infecciosas, devido este indicador também não ocorrer de forma homogênea no país, principalmente na Região Norte, onde a pobreza aumentou nos últimos anos. O Índice de Desenvolvimento Humano Municipal (IDHM) é uma evolução metodológica do IDH, que aborda as mesmas três dimensões deste indicador (renda, longevidade e educação). Sendo que, o IDHM também considera informações relacionadas às realidades socioeconômicas dos municípios brasileiros (PNUD, 2016).

Este desafio tem sido observado na necessidade de entender a distribuição das hepatites B e C, que devido não ocorrerem de forma homogênea no território nacional, dificulta a avaliação de políticas públicas voltadas para o controle destas doenças. Neste contexto, a análise espacial de dados tem sido utilizada, nas últimas décadas, de forma expressiva em estudos epidemiológicos devido à mesma permitir a caracterização da distribuição geográfica de doenças e seus fatores de risco, além da abrangência territorial de políticas públicas, como por exemplo, os municípios adscritos nas áreas de abrangência dos Centros Regionais de Saúde (CRS).

Os CRS são unidades técnicas e administrativas, que fazem parte da Secretaria Estadual de Saúde do Pará (SESPA) e são responsáveis pela execução de ações relacionadas às políticas públicas de saúde do estado, tais como, a cobertura de assistência básica em saúde, serviços de diagnósticos e campanhas de educação em saúde (GONÇALVES et al., 2016; PALÁCIOS et al., 2013; SESPA, 2016). Assim sendo, este estudo objetivou analisar a distribuição espacial de casos notificados de hepatites B e C, no Estado do Pará, relacionados com o IDHM dos municípios adstritos em três CRS, procurando contribuir para o estabelecimento de uma memória epidemiológica, sistemática e processual da doença.

\section{METODOLOGIA}

A população deste estudo descritivo e transversal foi de 1.458 casos notificados de hepatites $\mathrm{B}$ e $\mathrm{C}$, no período de 2010 a 2014, no $1^{\underline{a}}, 2^{\underline{a}}$ e $3^{a}$ CRS do estado do Pará. Os municípios que fazem parte do $1^{\text {a }}$ CRS são Belém, Ananindeua, Benevides, Marituba e Santa Bárbara do Pará. Os municípios que compõem o $2^{\text {a }}$ CRS são Santa Izabel do Pará, Acara, Bujaru, Colares, Concórdia do Pará, Santo Antônio do Tauá, São Caetano de Odivelas, Tomé Açú e Vigia de Nazaré. Os municípios que integram o $3^{a}$ CRS são Castanhal, Curuçá, Igarapé-Açú, Inhangapi, Magalhães Barata, Maracanã, Marapanim, São Francisco do Pará, São Domingos do Capim, São João da Ponta e Terra Alta.

As variáveis epidemiológicas analisadas foram: gênero (masculino ou feminino), faixa etária (criança se até 11; adolescente de 12 a 18; adulto de 19 a 59 e idoso com 60 anos ou mais), etnia (amarela, branca, indígena, parda ou preta), escolaridade (analfabeto, ensino fundamental, ensino médio e superior) e zona de moradia (urbana ou rural).

As variáveis relacionadas a contato interpessoal, procedimento invasivo percutâneo e tratamentos invasivos foram: contato sexual com portador de HBV e HCV, contato domiciliar com portador de HBV e HCV, três ou mais parceiros sexuais, doença sexualmente transmissível, uso de tatuagem ou piercing, acidente com material biológico, drogas inaláveis ou crack, acupuntura, transfusão de sangue, drogas injetáveis e medicamento injetável, tratamento cirúrgico, tratamento dentário, hemodiálise, transplante. Os dados destas variáveis foram obtidos nas fichas de Investigação de Hepatites Virais do SINAN, disponibilizadas pela SESPA. 
Análise espacial e epidemiológica de hepatites $\mathrm{B}$ e $\mathrm{C}$ e índice de desenvolvimento humano municipal, no estado do Pará
Nelson Veiga Gonçalves

Dayseanne Costa Vieira

Claudia do Socorro Carvalho Miranda

Vera Regina da Cunha Menezes Palácios

Simone Beverly Nascimento da Costa

Juan Andrade Guedes

Bruno de Oliveira Santos

Rodrigo Junior Farias da Costa

Sheila Cristina Martins e Silva

Rafael Aleixo Coelho de Oliveira

O levantamento dos dados cartográficos, populacionais e do IDHM dos municípios ocorreu no Sistema de Referência Geocêntrica para as Américas e no Censo Demográfico do Brasil pelo Instituto Brasileiro de Geografia e Estatística (IBGE, 2010; 2017).

Os dados do IDHM foram estratificados em cinco categorias: a muito baixo $(0$ a 0,49$)$, baixo $(0,5$ a 0,59$)$, médio $(0,6$ a 0,69$)$, alto $(0,7$ a 0,79$)$ e muito alto $(>0,8)$, de acordo com o Atlas de Desenvolvimento Humano (2010). Estes dados foram disponibilizados pelo IBGE, sendo os mesmos de domínio público e restrito.

Posteriormente, foi realizada a depuração das bases de dados obtidas, para retirar os registros com incompletudes e inconsistências das mesmas. Em seguida, estes dados foram georreferenciados, visando à implementação de um Banco de Dados Geográfico (BDGeo). Estes processos foram executados com os softwares TabWin 36b e Arcgis 10.2.

O cálculo das Taxas de Incidência (TI) Média das hepatites B e C, nos municípios dos três CRS estudados, teve como referência o indicador de endemicidade, utilizado pela Secretaria de Vigilância em Saúde (SVS), do Ministério da Saúde ${ }^{12}$, sendo que para este cálculo foi considerado como denominador a população média dos cinco anos do período do estudo (2010 a 2014), conforme a fórmula:

$$
\mathrm{TI}(\mathrm{HBV} \text { e HCV })=\frac{(\text { Média do no de casos com HBV e HCV })}{\text { Média população (2010 a 2014) }} \times 100.000
$$

$\mathrm{Na}$ sequência, foi realizada a análise das Taxas de Incidência Média (HBV e HCV) geradas utilizando o indicador preconizado pela OMS (2010), cujo cálculo ocorreu com a divisão das Taxas de Incidência Média dos municípios em quartis, que foram classificados em Baixa (até 2,34), Média (de 2,35 a 3,39), Alta (de 3,40 a 6,94) e Muito Alta (acima de 6,94), sendo os mesmos associados às cores verde-claro, amarela, laranja e vermelha, respectivamente, em conformidade com o padrão cartográfico nacional, para que fosse possível a observação da sua distribuição, por meio de mapas coropléticos. Os municípios que não registraram casos foram categorizados com ausência de casos e expressos na cor verde-escuro.

O interpolador de densidade Kernel foi utilizado para gerar a expressão visual da densidade de casos de infecção por HBV e HCV nos CRS estudados, devido o mesmo ser uma técnica de estatística espacial utilizada para analisar distribuição de dados.

O índice (I) de Moran Global Bivariado foi utilizado para avaliar a autocorrelação espacial entre as variáveis taxa de incidência e IDHM, considerando os municípios adstritos nos três CRS estudados, que ao serem variáveis espacializadas, admitem hipóteses de autocorrelação "negativa" ou "inversa" $(I<0)$, "aleatoriedade" $(I=0)$ e "positiva" ou "direta" $(I>0)$. Neste estudo, foi considerado correlação fraca quando o valor do índice I estiver próximo ao valor "0" (-0,5 a $0,5)$ e forte para valores próximos de "-1" $(<-0,5)$ e "1" $(>0,5)$. Com significância estatística espacial para um $\mathrm{p}$-valor $<0,05$.

Para a análise estatística dos dados relacionados às variáveis do indivíduo foi utilizado o teste não paramétrico do qui-quadrado de proporções esperadas iguais (teste de aderência, para modelos uniformes de probabilidade), com $\mathrm{p}<0,05$.

Este trabalho procurou garantir as exigências éticas, obedecendo à Declaração de Helsinque, ao Código de Nuremberg e às normas da Resolução № 466/12 do Conselho Nacional de Saúde, sendo que o mesmo recebeu parecer favorável para sua realização com o número 1.095.773/2015, do Comitê de Ética em Pesquisa da Universidade do Estado do Pará.

\section{RESULTADOS}

Foram georreferenciados 1.458 casos notificados de hepatites B e C, no período de 2010 a 2014 , distribuídos nos municípios adstritos nos $1^{\underline{a}}, 2^{\underline{a}}$ e $3^{\underline{a}}$ CRS do estado do Pará. A análise da série 
Análise espacial e epidemiológica de hepatites $\mathrm{B}$ e $C$ e índice de desenvolvimento humano municipal, no estado do Pará
Nelson Veiga Gonçalves

Dayseanne Costa Vieira

Claudia do Socorro Carvalho Miranda

Vera Regina da Cunha Menezes Palácios

Simone Beverly Nascimento da Costa

Juan Andrade Guedes

Bruno de Oliveira Santos

Rodrigo Junior Farias da Costa

Sheila Cristina Martins e Silva

Rafael Aleixo Coelho de Oliveira

histórica mostrou que o $1^{\text {a }}$ CRS apresentou o maior número de casos $(87 \%)$ e o $2^{\text {a }}$ CRS o menor (4\%). Sendo que, os $2^{a}$ e $3^{\text {a }}$ CRS apresentaram de forma geral diminuição no número de notificações. A maior taxa de incidência foi observada no $1^{\text {a }}$ CRS que se manteve acima da média, exceto no ano de 2011. Neste CRS também foi identificado um aumento no número de casos nos anos de 2012 e 2014. O 2 ${ }^{a}$ CRS apresentou a menor taxa de incidência do período, conforme a figura 1 .

Figura 1: Série Histórica das hepatites $B$ e $C$ dos municípios do $1^{\circ}, 2^{\circ}$ e $3^{\circ} \mathrm{CRS}$, no período de 2010 a 2014, Pará, Brasil.

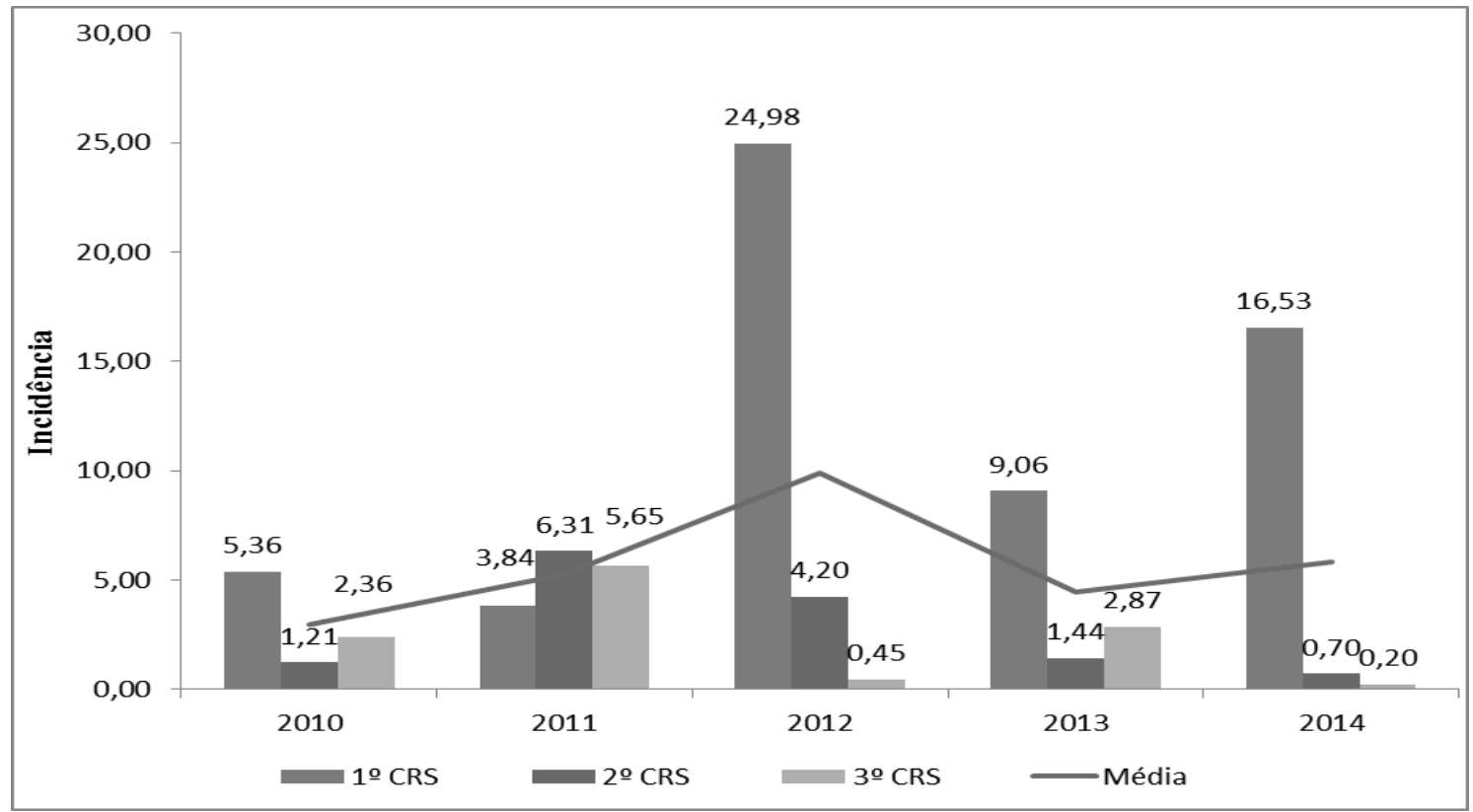

Fonte: EPIGEO/DSCM/CCBS/UEPA, 2017.

$\mathrm{Na}$ totalidade dos casos, o maior percentual dos casos notificados das doenças, ao longo da série estudada, ocorreu em indivíduos do gênero masculino com $58.9 \%$, na idade adulta (19 a 59 anos) com $65.6 \%$, na etnia parda com $65.1 \%$, com escolaridade ensino fundamental ( $5^{\mathrm{a}}$ a $8^{\mathrm{a}}$ série) com $27.3 \%$, e que residiam em zona urbana com 93,0\%. Esses resultados, exceto o gênero, mostraram-se significantes em relação aos casos notificados, conforme pode observado na Tabela 1:

A análise conjunta para os três CRS dos fatores de riscos comportamentais e biológicos mostrou que os procedimentos invasivos percutâneos relacionados à utilização de medicamentos injetáveis estão entre os maiores percentuais. Contudo, no $1^{\circ}$ a ocorrência de doença sexualmente transmissível (31\%) foi a variável que apresentou o maior percentual. Já no $2^{\circ}$ e $3^{\circ}$ CRS, o fator de risco de maior percentual foram medicamentos injetáveis com $29 \%$ e $36 \%$ respectivamente. Esses resultados foram significativos estatisticamente, conforme a tabela 2. 
Análise espacial e epidemiológica de hepatites B e $\mathrm{C}$ e índice de desenvolvimento humano municipal, no estado do Pará
Nelson Veiga Gonçalves

Dayseanne Costa Vieira

Claudia do Socorro Carvalho Miranda

Vera Regina da Cunha Menezes Palácios

Simone Beverly Nascimento da Costa

Juan Andrade Guedes

Bruno de Oliveira Santos

Rodrigo Junior Farias da Costa

Sheila Cristina Martins e Silva

Rafael Aleixo Coelho de Oliveira

Tabela 1: Perfil epidemiológico das hepatites B e $C$ entre os anos de 2010 a $2014(n=1.458)$ nos municípios do 1ำ 2ํㅡ e 3ํㅡㄴ, Pará, Brasil.

\begin{tabular}{|c|c|c|c|c|c|c|c|c|c|c|c|c|c|}
\hline \multirow{2}{*}{ Variáveis } & \multicolumn{2}{|c|}{2010} & \multicolumn{2}{|c|}{2011} & \multicolumn{2}{|c|}{2012} & \multicolumn{2}{|c|}{2013} & \multicolumn{2}{|c|}{2014} & \multicolumn{2}{|c|}{ Total } & \multirow{2}{*}{ P-Valor } \\
\hline & $\mathbf{n}$ & $\%$ & $\mathbf{n}$ & $\%$ & $\mathbf{n}$ & $\%$ & $\mathbf{n}$ & $\%$ & $\mathbf{n}$ & $\%$ & $\mathbf{n}$ & $\%$ & \\
\hline \multicolumn{14}{|l|}{ Gênero } \\
\hline Feminino & 140 & 38,9 & 138 & 51,1 & 76 & 28,2 & 108 & 46,2 & 133 & 41.1 & 595 & 41,1 & \multirow{2}{*}{0,0918} \\
\hline Masculino & 220 & 61,1 & 132 & 48,9 & 194 & 71,9 & 126 & 53,9 & 191 & 59.0 & 863 & 58,9 & \\
\hline \multicolumn{14}{|c|}{ 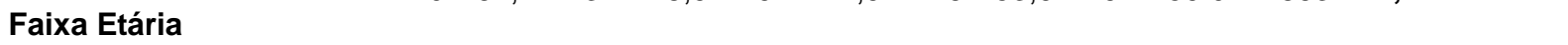 } \\
\hline Até 11 anos & 20 & 4,2 & 24 & 8,9 & 16 & 5,9 & 7 & 3,0 & 4 & 1,2 & 71 & 4,6 & \multirow{4}{*}{$<0,0001$} \\
\hline 12 a 18 anos & 15 & 5,6 & 30 & 11,1 & 15 & 5,6 & 14 & 6,0 & 67 & 20,7 & 141 & 9,8 & \\
\hline 19 a 59 anos & 238 & 66,1 & 179 & 66,3 & 200 & 74,1 & 169 & 72,2 & 159 & 49,1 & 945 & 65,6 & \\
\hline 60 anos ou mais & 87 & 24.2 & 37 & 13,7 & 39 & 14,4 & 44 & 18,8 & 94 & 29,0 & 301 & 20,0 & \\
\hline \multicolumn{14}{|l|}{ Etnia } \\
\hline Amarela & 2 & 0,6 & 1 & 0,4 & 1 & 0,4 & 0 & 0,0 & 0 & 0,0 & 4 & 0,3 & \multirow{6}{*}{$<0,0001$} \\
\hline Branca & 34 & 9,4 & 24 & 8,9 & 8 & 3,0 & 19 & 8,1 & 15 & 4,6 & 100 & 6,8 & \\
\hline Indígena & 1 & 0,3 & 2 & 0,8 & 1 & 0,4 & 14 & 6,0 & 54 & 16,7 & 72 & 4,8 & \\
\hline Parda & 227 & 63,1 & 225 & 83,3 & 140 & 51,9 & 139 & 59,4 & 220 & 67,9 & 951 & 65,1 & \\
\hline Preta & 10 & 2,8 & 11 & 4,1 & 58 & 21,5 & 12 & 5,1 & 10 & 3,1 & 101 & 7,3 & \\
\hline Ignorado & 86 & 23,9 & 7 & 2,6 & 62 & 23,0 & 50 & 21,4 & 25 & 7,7 & 230 & 15,7 & \\
\hline \multicolumn{14}{|l|}{ Escolaridade } \\
\hline Analfabeto & 3 & 0,8 & 6 & 2,2 & 2 & 0,7 & 5 & 2,1 & 8 & 2,5 & 24 & 1,7 & \multirow{5}{*}{$<0,0001$} \\
\hline E. Fundamental & 93 & 25,8 & 128 & 47,4 & 56 & 20,7 & 60 & 25,6 & 55 & 17,0 & 392 & 27,3 & \\
\hline E. Médio & 77 & 21,4 & 51 & 18,9 & 30 & 11,1 & 31 & 13,3 & 29 & 9,0 & 218 & 14,7 & \\
\hline E. Superior & 20 & 5,6 & 10 & 3,7 & 3 & 1,1 & 10 & 4,3 & 9 & 2,8 & 52 & 3,5 & \\
\hline Ignorado & 167 & 46,4 & 75 & 27,8 & 179 & 66,3 & 128 & 54,7 & 223 & 68,8 & 772 & 52,8 & \\
\hline \multicolumn{14}{|c|}{ - } \\
\hline Urbana & 351 & 97,5 & 241 & 89,3 & 261 & 96,7 & 203 & 86,8 & 307 & 94,8 & 1363 & 93,0 & \multirow{2}{*}{$<0,0001$} \\
\hline Rural & 9 & 2,5 & 29 & 10,7 & 9 & 3,3 & 31 & 13,3 & 17 & 5,3 & 95 & 7,0 & \\
\hline
\end{tabular}

Fonte: EPIGEO/DSCM/CCBS/UEPA, 2017.

Tabela 2: Fatores de risco comportamentais e biológicos das hepatites B e C por CRS ( $n=100 \%)$,

Pará, Brasil.

\begin{tabular}{|c|c|c|c|c|c|c|}
\hline Fatores de risco & $\begin{array}{c}\text { 1ㄹ }^{\mathrm{a}} \mathrm{CRS} \\
(\%)\end{array}$ & P-Valor ${ }^{(1)}$ & $\begin{array}{c}\text { 2-CRS }^{\text {a CRS }} \\
(\%)\end{array}$ & P-Valor ${ }^{(1)}$ & $\begin{array}{c}\text { 3-CRS }^{\text {a CRS }} \\
(\%)\end{array}$ & P-Valor ${ }^{(1)}$ \\
\hline $\begin{array}{l}\text { Contato sexual com portador de } \\
\mathrm{HBV} / \mathrm{HCV}\end{array}$ & 7 & & 2 & & 9 & \\
\hline Três ou mais parceiros sexuais & 4 & & 8 & & 13 & \\
\hline Doença sexualmente transmissível & 31 & & 22 & & 8 & \\
\hline Tatuagem / Piercing & 5 & & 7 & & 11 & \\
\hline Acupuntura & 2 & & 0 & & 3 & \\
\hline Drogas ilícitas injetáveis & 3 & & 3 & & 0 & \\
\hline Acidente com material biológico & 4 & $<0,0001$ & 3 & $<0,0001$ & 2 & $<0,0001$ \\
\hline Transfusão de sangue & 2 & & 2 & & 3 & \\
\hline Medicamento injetável & 21 & & 29 & & 36 & \\
\hline Tratamento Cirúrgico & 11 & & 8 & & 11 & \\
\hline Tratamento dentário & 6 & & 16 & & 4 & \\
\hline Hemodiálise & 2 & & 0 & & 0 & \\
\hline Transplante & 2 & & 0 & & 0 & \\
\hline Total & 100 & & 100 & & 100 & \\
\hline
\end{tabular}

Fonte: EPIGEO/DSCM/CCBS/UEPA, 2017. 
Análise espacial e epidemiológica de hepatites B e $\mathrm{C}$ e índice de desenvolvimento humano municipal, no estado do Pará
Nelson Veiga Gonçalves

Dayseanne Costa Vieira

Claudia do Socorro Carvalho Miranda

Vera Regina da Cunha Menezes Palácios

Simone Beverly Nascimento da Costa

Juan Andrade Guedes

Bruno de Oliveira Santos

Rodrigo Junior Farias da Costa

Sheila Cristina Martins e Silva

Rafael Aleixo Coelho de Oliveira

A análise da relação espacial, considerando os municípios contidos na área de estudo mostrou que no $1^{\circ}$ CRS, o parâmetro da taxa de incidência foi considerado muito alto $(60 \%)$ e o do IDHM médio $(60 \%)$. Sendo que $100 \%$ dos municípios notificaram casos. O índice (I) de Moran Global Bivariado, conforme a metodologia mostrou forte autocorrelação espacial inversa entre estas duas variáveis $(l=-0,7037)$.

No $2^{\circ}$ CRS, o parâmetro da taxa de incidência foi médio (45\%) e os do IDHM foram médios $(57 \%)$ e baixos (43\%). Sendo que $22 \%$ dos municípios não notificaram casos. O índice (I) de Moran Global Bivariado mostrou uma fraca autocorrelação espacial inversa entre estas duas variáveis $(l=-0,0549)$.

No $3^{\text {a }}$ CRS, o parâmetro da taxa de incidência foi baixo (27\%) e do IDHM baixo (83\%). Sendo que $46 \%$ dos municípios não notificaram casos. O índice (I) de Moran Global Bivariado mostrou uma fraca autocorrelação espacial inversa entre estas duas variáveis $(l=-0,0070)$, conforme a figura 2 .

Figura 2: IDHM e Incidência de casos das hepatites B e C nos municípios do $1^{\circ}, 2^{\circ}$ e $3^{\circ} \mathrm{CRS}$, no período de 2010 a 2014, Pará, Brasil.

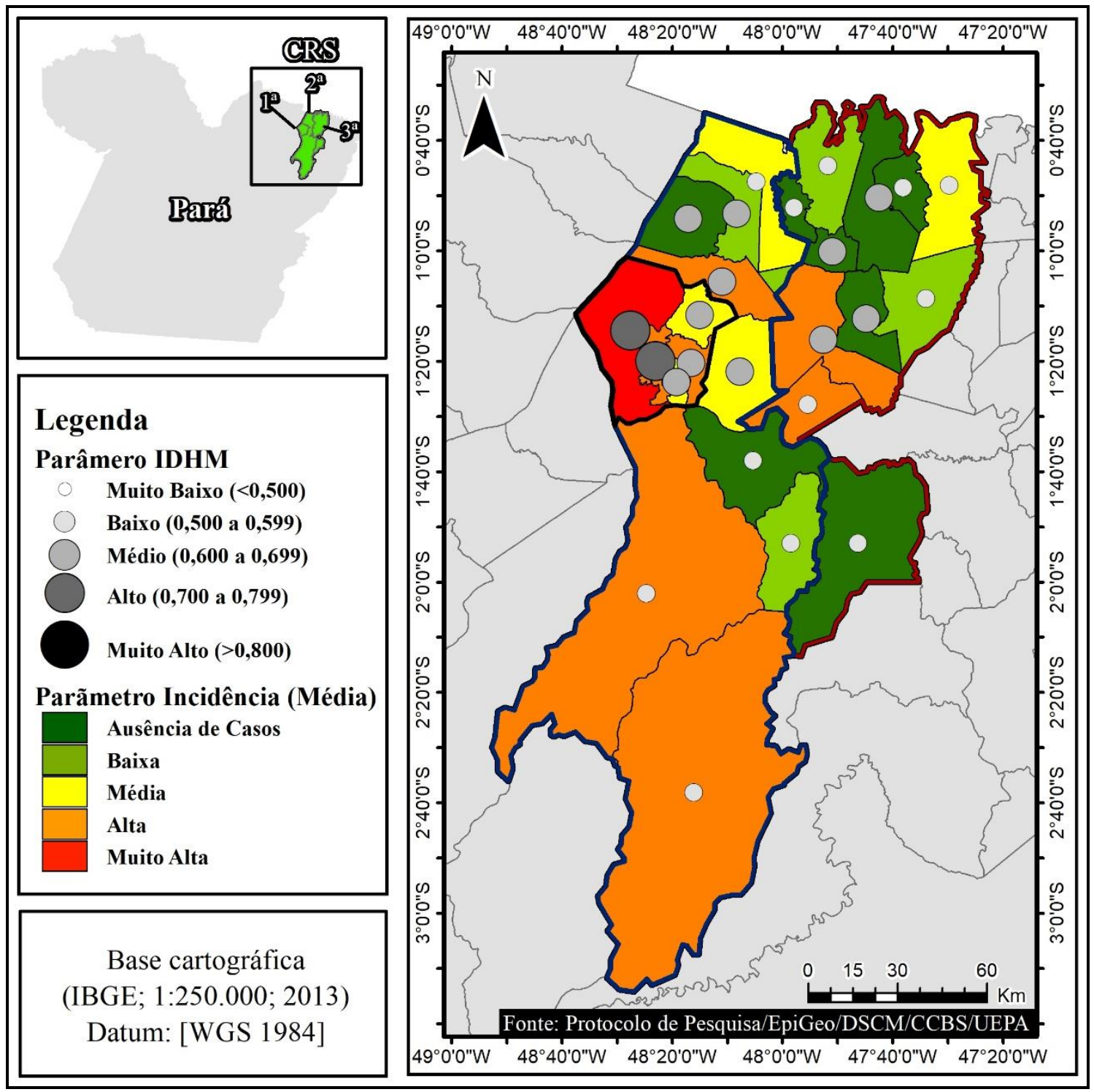

Fonte: EPIGEO/DSCM/CCBS/UEPA, 2017. 
Análise espacial e epidemiológica de hepatites B e $\mathrm{C}$ e índice de desenvolvimento humano municipal, no estado do Pará
Nelson Veiga Gonçalves

Dayseanne Costa Vieira

Claudia do Socorro Carvalho Miranda

Vera Regina da Cunha Menezes Palácios

Simone Beverly Nascimento da Costa

Juan Andrade Guedes

Bruno de Oliveira Santos

Rodrigo Junior Farias da Costa

Sheila Cristina Martins e Silva

Rafael Aleixo Coelho de Oliveira

As análises de distribuição espacial de casos de hepatite $B$ e $C$ realizadas com a interpolação de Kernel evidenciaram que no $1^{\circ}$ CRS os municípios de Belém e Ananindeua apresentaram uma densidade muito alta de casos, caracterizando um aglomerado. Enquanto que no $2^{\circ}$ e $3^{\circ}$ CRS foi observada uma baixa densidade dos mesmos, em todos os municípios, conforme pode ser observado na Figura 3.

Figura 3: Densidade de casos das hepatites B e C, nos municípios do $1^{\circ}, 2^{\circ}$ e $3^{\circ}$ CRS, no período de 2010 a 2014, Pará, Brasil.

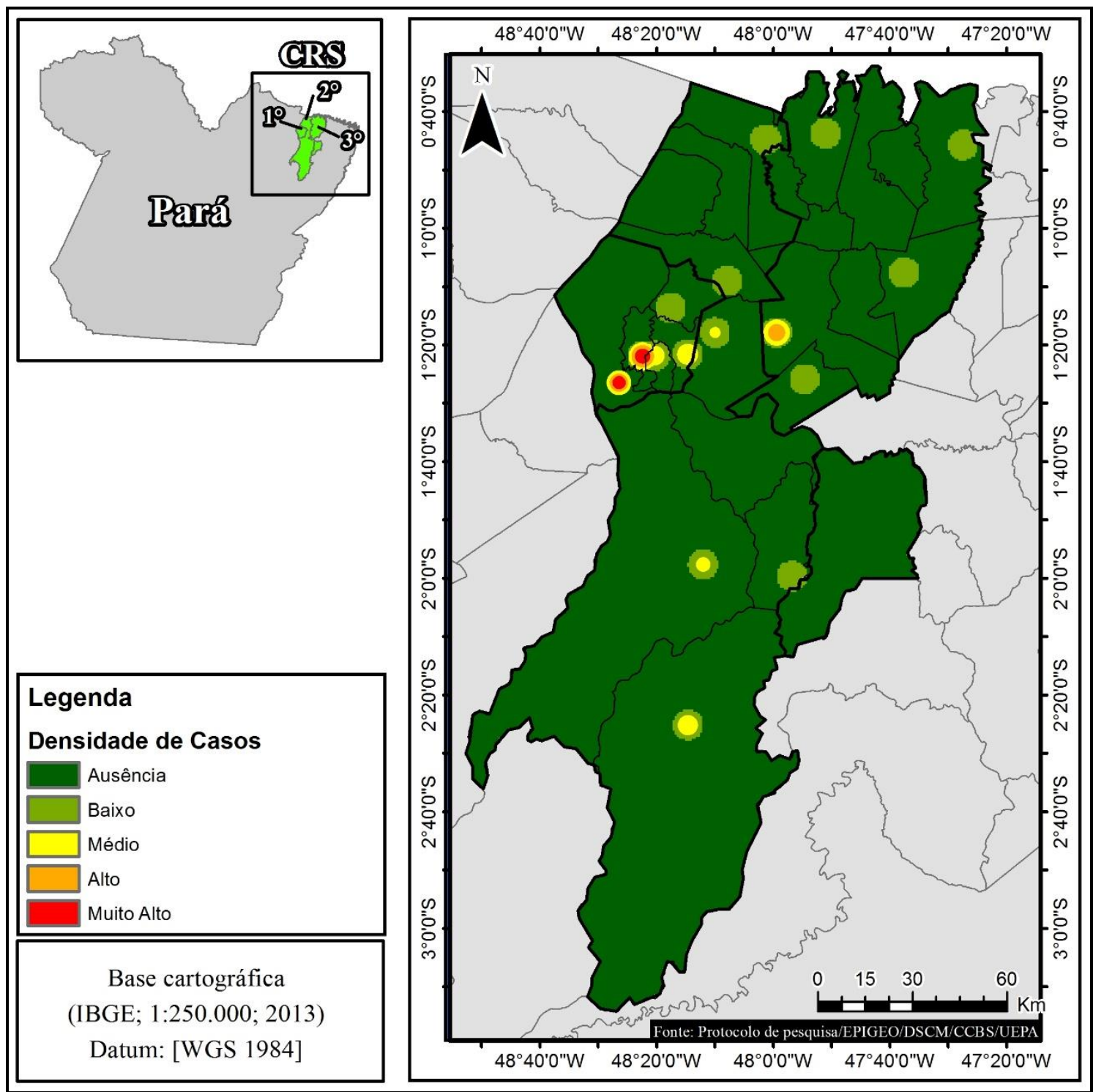

Fonte: EPIGEO/DSCM/CCBS/UEPA, 2017.

\section{DISCUSSÃO}

A análise do número de casos notificados de hepatite $B$ e $C$ mostrou uma grande disparidade entre os percentuais de notificação destas doenças na área de estudo, sendo que o $1^{\circ}$ CRS notificou mais 
Análise espacial e epidemiológica de hepatites B e $\mathrm{C}$ e índice de desenvolvimento humano municipal, no estado do Pará
Nelson Veiga Gonçalves

Dayseanne Costa Vieira

Claudia do Socorro Carvalho Miranda

Vera Regina da Cunha Menezes Palácios

Simone Beverly Nascimento da Costa

Juan Andrade Guedes

Bruno de Oliveira Santos

Rodrigo Junior Farias da Costa

Sheila Cristina Martins e Silva

Rafael Aleixo Coelho de Oliveira

casos. Este fato pode ser justificado devido o mesmo abranger cinco municípios, entre os quais a capital do estado (Belém) e um município de grande porte (Ananindeua), que apresentam um grande contingente populacional (IBGE, 2010). Contudo, estes dados também podem estar relacionados à maior oferta de serviços de saúde, tais como, diagnóstico e acompanhamento de casos.

Foi observado um decréscimo no número de notificações de hepatites $B$ e $C$ no $2^{\circ}$ e $3^{\circ}$ CRS. Este fato pode estar relacionado à ocorrência de subnotificações devido à ausência de políticas de acompanhamento e prevenção desse agravo, de forma contínua e processual, nos municípios que compõe estas unidades territoriais de análise.

Ainda no $1^{\circ}$ CRS foi identificado que em 2012 e 2014 correram aumentos das notificações nestes anos. Este fato pode ser justificado pela execução de políticas de acompanhamento e controle de hepatites virais, com a realização de campanhas de busca ativa de casos, tratamento da doença e de ações educativas ocorridas nos municípios deste território, em conformidade com as politicas públicas preconizado pelo Ministério da Saúde (BRASIL, 2005).

No que diz respeito às variáveis relacionadas ao indivíduo foi observado predominância da infecção por hepatite $\mathrm{B}$ e $\mathrm{C}$ no sexo masculino. Este fato pode estar relacionado a fatores comportamentais como a prática de atividade sexual sem uso de preservativo (CARVALHO et al., 2014; DIOGO et al., 2012; VIEIRA; VENTURA; SOUSA, 2014).

Na maioria dos casos notificados foi declarada a etnia parda, que pode estar relacionado à formação étnica no Brasil, sobretudo na Amazônia, onde a presença de pardos nesta região é devida a miscigenação entre brancos, negros e índios. De acordo com o IBGE (2010), dos 191 milhões de brasileiros entrevistados, 82 milhões foram autodeclarados como pardos, passando as populações negras e pardas a ser considerada a maioria no Brasil.

A análise da variável faixa etária mostrou que os casos de hepatite $B$ e $C$, na área de estudo, foram mais incidentes em adultos em idade produtiva. Este fato pode impactar a economia dos municípios, tornando a ocorrência dessas doenças, um grande problema de saúde pública (CASTELO et al., 2007).

Diversos fatores podem contribuir para o diagnóstico tardio dessas doenças nesta faixa etária, tais como o longo período de incubação dos vírus e a ausência de sinais e sintomas, dado o caráter silencioso destes tipos de hepatites (MARGREITER et al., 2015). Além de aspectos comportamentais adquiridos ao longo da vida, tais como atividade sexual de risco, uso de drogas ilícitas injetáveis e outras exposições a sangue e hemoderivados, fato este que demonstra a produção social destas doenças (ALAVIAN; TAHERI, 2012; AQUINO, J. A. et al., 2008; BENSABATH; LEÃO, 2003; BRASIL, 2005; 2010; COSTA JÚNIOR et al., 2013; DIOGO et al., 2012; OLIVEIRA et al., 2005; OLIVEIRA; FOCACCIA, 2010;)

A maioria dos casos notificados apresentou baixa escolaridade com ensino fundamental. Esta situação pode estar relacionada às condições socioeconômicas desfavoráveis, e que podem favorecer a transmissão da doença (ALVES et al., 2014; BRASIL, 2010).

Com relação à zona de residência o maior número de casos ocorreu na área urbana. Esta associação pode ter ocorrido em função da alta densidade populacional nesta área, (IBGE, 2010). Outro fator que pode ter contribuído para a maior notificação de casos pode ter sido a maior oferta e acesso a exames laboratoriais, voltados para diagnóstico (PUDELCO; KOEHLER; BISETTO, 2014).

Com relação aos fatores de risco comportamentais e biológicos duas variáveis apresentaram as maiores significâncias estatísticas, nas três áreas de estudo. A primeira foi ocorrência de doenças sexualmente transmissíveis, corroborando com estudos que relatam a possibilidade de associação desta com as hepatites $\mathrm{B}$ e $\mathrm{C}$, em um mesmo indivíduo, devido à semelhança nas formas de transmissão destas doenças (BRASIL, 2017; CAVALHEIRO, 2007; TERRAULT, 2002). Esta situação, ao ser considerada a realidade dos municípios do estado do Pará aponta para a necessidade de intensificação de políticas públicas de saúde, tais como, campanhas de educação e promoção à 
Análise espacial e epidemiológica de hepatites B e $\mathrm{C}$ e índice de desenvolvimento humano municipal, no estado do Pará
Nelson Veiga Gonçalves

Dayseanne Costa Vieira

Claudia do Socorro Carvalho Miranda

Vera Regina da Cunha Menezes Palácios

Simone Beverly Nascimento da Costa

Juan Andrade Guedes

Bruno de Oliveira Santos

Rodrigo Junior Farias da Costa

Sheila Cristina Martins e Silva

Rafael Aleixo Coelho de Oliveira

saúde, onde através de práticas educativas pode ocorrer por meio de práticas educativas podem possibilitar a diminuição do número de casos com este tipo de associação.

A segunda variável foi uso de medicamentos injetáveis, que enquanto fator de risco pode estar associado à falta de perícia com relação à prática adequada de assepsia dos equipamentos utilizados em procedimentos cirúrgicos de baixa e média complexidade (ALTER, 2002; KUBOCA, 2010). Esta situação tem sido observada também, de forma recorrente, na área objeto deste estudo, onde a prática de aplicação de injetáveis e pequenos procedimentos cirúrgicos ainda é realizada em residências, principalmente nas áreas rurais e ribeirinhas, onde existe a carência de profissionais e serviços de saúde.

A autocorrelação espacial inversa observada entre os parâmetros do IDHM e da incidência, utilizando o índice (I) de Moran nos três CRS estudados, pode estar relacionada às diferentes situações observadas, tais como, renda, longevidade, educação e cobertura de políticas públicas em saúde. No caso do $1^{\circ} \mathrm{CRS}$ ocorreu uma forte autocorrelação espacial inversa $(\mathrm{I}=-0,7037)$, possivelmente devido $100 \%$ dos municípios que compõem este CRS terem notificado casos, sendo que na maioria dos municípios deste CRS foram observados indiciadores baixos de educação da população, pois em locais com déficit educacional são observadas alta incidência de doenças infecciosas, dentre as quais as hepatites virais (KUBOTA; CAMPOS; PEREIRA, 2014).

No $2^{\circ}$ CRS foi observada uma fraca autocorrelação espacial inversa $(I=-0,0549)$, possivelmente devido a $22 \%$ dos municípios que compõem este CRS não terem notificado casos, este fato pode indicar um silêncio epidemiológico, com possíveis falhas nos serviços de busca ativa dos casos (KUBOTA; CAMPOS; PEREIRA, 2014; PAROLIN et al., 2013; XIMENES et al., 2010). A autocorrelação espacial inversa pode estar relacionada à baixa renda, identificada na maioria dos municípios que compõem este CRS, uma vez que esta pode implicar na fragilidade socioeconômica de grande parte de sua população que vive, sobretudo, em aglomerados ou adensamentos populacionais, como invasões e assentamentos, com ausência de serviços públicos essenciais, tais como os de promoção à saúde (BENSABATH; LEÃO, 2003; FERREIRA; SILVEIRA, 2004).

O 3 a $\mathrm{CRS}$ também apresentou uma fraca autocorrelação espacial inversa $(I=-0,0070)$, possivelmente devido $46 \%$ dos municípios que compõem este CRS não terem notificado casos, que mostra a ocorrência de um expressivo silêncio epidemiológico neste território, que pode estar associada a áreas sem cobertura de serviços essenciais de saúde (KUBOTA; CAMPOS; PEREIRA, 2014; XIMENES et al., 2010).

Este cenário epidemiológico reflete a precariedade das condições socioeconômicas de grande parte das populações residentes neste território, onde foram verificados baixos indicadores de escolaridade e renda na maioria dos seus municípios, indicando a existência de áreas com grande vulnerabilidade social e possibilidade de uma maior exposição aos possíveis fatores de riscos da doença (FERREIRA; SILVEIRA, 2004; XIMENES et al., 2010).

O aglomerado de casos observados, a partir da utilização da técnica de Kernel, em Belém e Ananindeua, municípios estes assistidos pelo $1^{\circ} \mathrm{CRS}$, pode estar relacionado à execução de políticas públicas de saúde voltadas para acompanhamento e controle das hepatites virais, de forma processual. Este fato não foi observado em outros municípios adscritos nas áreas de abrangência dos demais CRS. Esta situação corrobora com estudos realizados por Kassouf (KASSOUF, 2005); Morais, Rodrigues e Gerhardt (2008) e World Bank (2006) que ao fazerem comparações entre serviços de saúde prestados, em áreas metropolitanas, verificaram que as mesmas apresentam maior cobertura de serviços de saúde.

\section{CONCLUSÃO}

A notificação das hepatites $B$ e $C$ reflete um grande problema de saúde pública nos municípios paraenses integrantes das áreas estudadas. Pois, no perfil socioepidemiológico foi evidenciada a 
Análise espacial e epidemiológica de hepatites B e $\mathrm{C}$ e índice de desenvolvimento humano municipal, no estado do Pará
Nelson Veiga Gonçalves

Dayseanne Costa Vieira

Claudia do Socorro Carvalho Miranda

Vera Regina da Cunha Menezes Palácios

Simone Beverly Nascimento da Costa

Juan Andrade Guedes

Bruno de Oliveira Santos

Rodrigo Junior Farias da Costa

Sheila Cristina Martins e Silva

Rafael Aleixo Coelho de Oliveira

ocorrência em indivíduos com baixa escolaridade, adultos, pardos e residentes em área urbana, sendo as DSTs e os medicamentos injetáveis fatores de risco mais significantes.

Foi verificada uma dependência espacial entre a distribuição das hepatites estudadas e as variáveis socioeconômicas nos territórios analisados, refletindo uma autocorrelação inversa entre estas variáveis. Assim, a baixa escolaridade e a baixa renda mostraram-se relevantes enquanto fatores de risco associados à transmissão dos agravos estudados, mostrando aglomerados de casos em regiões com iniquidades sociais, mesmo em municípios com IDHM alto, evidenciando gradientes de produção socioeconômicos destas hepatites.

Tais achados apontaram para a necessidade de adequação de intervenções, de acordo com as necessidades específicas dos territórios, bem como a priorização de ações em saúde, objetivando a redução de fatores de risco, em áreas com maior suscetibilidade a transmissão. Contudo, a elevada subnotificação de dados relacionados às hepatites virais observadas, demonstra falhas no sistema e oportunidades perdidas para a realização de um diagnóstico da produção social destes agravos de forma mais precisa, além da possível ocorrência de silêncio epidemiológico.

Considerando as diferentes relações entre as variáveis estudadas as ferramentas computacionais utilizadas, na análise espacial dos dados, foram satisfatórias para a construção do cenário epidemiológico das hepatites estudadas. Desta forma, estas tecnologias demonstraram potencial para prover os gestores em saúde com informações voltadas para a vigilância contínua e sistemática dos agravos, que foram objeto deste estudo.

\section{AGRADECIMENTOS}

Agradecemos o apoio logístico do Laboratório de Epidemiologia e geoprocessamento da Amazônia (EPIGEO), do Centro de Ciências Biológicas e da Saúde (CCBS) da Universidade do Estado do Pará (UEPA).

Agradecemos o apoio logístico do $1^{\circ}, 2^{\circ}$ e $3^{\circ}$ Centro Regional de Saúde do Estado do Pará e da Secretaria de Saúde Pública do Estado do Pará.

\section{CONFLITO DE INTERESSES}

Este trabalho não apresentou conflitos de interesse.

\section{REFERÊNCIAS}

ALAVIAN, S. M.; TAHERI, S. A Global Perspective on the Intrafamilial Transmission of Hepatitis B Virus Infection. International Journal of Travel Medicine and Global Health, Irã, v. 3, n. 1, p. 1-4, 2012. Disponível em:

$<$ https://www.researchgate.net/publication/257983808_A_Global_Perspective_on_the_Intrafamilial_Tr ansmission_of_Hepatitis_B_Virus_Infection>. Acesso em: 20 jun. 2017.

ALVES, M. R. et al. Perfil epidemiológico dos casos de hepatite $C$ em uma diretoria regional de saúde da Bahia. Revista de Pesquisa: Cuidar é Fundametal, Rio de Janeiro, v. 6, n. 3, p. 1-7, 2014.

Disponível em: <http://www.seer.unirio.br/index.php/cuidadofundamental/article/view/3299/pdf_1327>. Acesso em: 12 mar. 2017.

AQUINO, J. A. et al. Soroprevalência de infecções por vírus da hepatite $B$ e vírus da hepatite $C$ em indivíduos do Estado do Pará. Revista da Sociedade Brasileira de Medicina Tropical, Uberaba, v. 41, p. 334-337, 2008. https://doi.org/10.1590/S0037-86822008000400003

ALTER, M. J. Preventionof spread of hepatitis C. Hepatology, New York, v. 36, n. 5, p. 1-5, 2002. Disponível em: <https://www.ncbi.nlm.nih.gov/pubmed/12407581>. Acesso em: 07 jun. 2017. https://doi.org/10.1053/jhep.2002.36389 
Análise espacial e epidemiológica de hepatites B e $\mathrm{C}$ e índice de desenvolvimento humano municipal, no estado do Pará
Nelson Veiga Gonçalves

Dayseanne Costa Vieira

Claudia do Socorro Carvalho Miranda

Vera Regina da Cunha Menezes Palácios

Simone Beverly Nascimento da Costa Juan Andrade Guedes

Bruno de Oliveira Santos

Rodrigo Junior Farias da Costa

Sheila Cristina Martins e Silva

Rafael Aleixo Coelho de Oliveira

BRASIL. Ministério da Saúde. Boletim Epidemiológico Hepatites Virais. Brasília: Ministério da Saúde, 2010.

BENSABATH, F.; LEÃO, R. N. Q. Epidemiologia na Amazônia Brasileira. In: FOCACCIA, R. Tratado das Hepatites Virais. São Paulo: Atheneu, 2003.

BRASIL. Ministério da Saúde. A, B, C, D, E de hepatites para comunicadores. 1 ed. Brasília: Ministério da Saúde, 2005.

. Ministério da Saúde. Programa Nacional para a Prevenção das Hepatites Virais. Brasília: Ministério da Saúde, 2005.

. Ministério da Saúde. Doenças infecciosas e parasitárias. Guia de bolso, 8 ed. Brasília: Ministério da Saúde, 2010.

. Ministério da Saúde. Informe da Atenção Básica. Brasília: Ministério da Saúde, 2010.

. Ministério da Saúde. Conselho Nacional de Saúde. Resolução № 466, de 12 de dezembro de 2012. Disponível em:

<http://bvsms.saude.gov.br/bvs/saudelegis/cns/2013/res0466_12_12_2012.html>. Acesso em: 07 jun. 2017.

. Ministério da Saúde. Protocolo Clínico e Diretrizes Terapêuticas para Hepatite C e Coinfecções. Brasília: Ministério da Saúde, 2017. 2017.

. Ministério da Saúde. Boletim epidemiológico: hepatites virais. Brasília: Ministério da Saúde,

CASTELO, A. et al. Estimativas de custo da hepatite crônica B no Sistema Único de Saúde brasileiro em 2005. Revista da Associação Medica Brasileira, São Paulo, v. 53, n.6, p. 486-491, 2007. https://doi.org/10.1590/S0104-42302007000600013

CARVALHO, J. R. et al. Método para estimação de prevalência de hepatites $B$ e $C$ crônicas e cirrose hepática - Brasil, 2008. Epidemiologia e Serviço Saúde, Brasília, v. 23, n. 4, p. 1-9. 2014.

Disponível em: <http://scielo.iec.pa.gov.br/pdf/ess/v23n4/v23n4a11.pdf>. Acesso em: 09 abr. 2017. https://doi.org/10.5123/S1679-49742014000400011

CAVALHEIRO, N. P. Sexual transmission of hepatitis C. Revista da Sociedade Brasileira de Medicina Tropical, Uberaba, v. 49, n. 5, p. 1-6, 2007. Disponível em:

<http://www.scielo.br/pdf/rimtsp/v49n5/a01v49n5.pdf>. Acesso em: 12 mar. 2017.

COSTA JÚNIOR, P. R. S. et al. Infecção por hepatites b e c em um município do estado do Pará.

Revista Paraense de Medicina, Belém, v. 27, n. 2, p. 1-10, 2013. Disponível em:

<http://files.bvs.br/upload/S/0101-5907/2013/v27n2/a3673.pdf>. Acesso em: 12 mar. 2017.

DIOGO, F. V. et al. Estudo da soroprevalência da infecção pelo vírus da hepatite B entre os doadores de sangue do Núcleo Hemoterápico da Santa Casa de Alfenas (Alfenas/MG) por meio do marcador anti-HBc. Revista Brasileira de Pesquisa em Saúde, Espirito Santo, v. 14, n. 2, p. 1-9,

2012. Disponível em: <http://periodicos.ufes.br/RBPS/article/view/4188/3312>. Acesso em: 09 abr. 2017.

FERREIRA, C. T.; SILVEIRA, T. R. Hepatites virais: aspectos da epidemiologia e da prevenção.

Revista Brasileira de Epidemiologia, Rio de Janeiro, v. 7, n. 4, p. 1-5, 2004. Disponível em: <http://www.scielo.br/pdf/rbepid/v7n4/10.pdf>. Acesso em: 17 jun. 2017.

https://doi.org/10.1590/S1415-790X2004000400010

GONÇALVES, N. V. et al. Distribuição espaço-temporal da leptospirose e fatores de risco em Belém, Pará, Brasil. Ciência e saúde coletiva, Rio de Janeiro, v. 21, n. 12, p. 1-9, 2016. Disponível em: <http://dx.doi.org/10.1590/1413-812320152112.07022016>. Acesso em: 25 ago. 2017.

https://doi.org/10.1590/1413-812320152112.07022016

IBGE. Instituto Brasileiro de Geografia e Estatística. SIRGAS. Rio de Janeiro: IBGE, 2017. 
Análise espacial e epidemiológica de hepatites B e $\mathrm{C}$ e índice de desenvolvimento humano municipal, no estado do Pará
Nelson Veiga Gonçalves

Dayseanne Costa Vieira

Claudia do Socorro Carvalho Miranda

Vera Regina da Cunha Menezes Palácios

Simone Beverly Nascimento da Costa Juan Andrade Guedes

Bruno de Oliveira Santos

Rodrigo Junior Farias da Costa

Sheila Cristina Martins e Silva

Rafael Aleixo Coelho de Oliveira

Instituto Brasileiro de Geografia e Estatística. Censo 2010. Rio de Janeiro: IBGE, 2010.

LOPES, T. G. S. L.; SCHINONI, M. I. Aspectos gerais da hepatite B. Revista de Ciências Médicas e Biologicas, Salvador, v. 10, n. 3, p. 1-8, 2011. Disponível em:

<https://portalseer.ufba.br/index.php/cmbio/article/view/5899/4251>. Acesso em: 18 jun. 2017.

https://doi.org/10.9771/cmbio.v10i3.5899

KASSOUF, A. L. Acesso aos serviços de saúde nas áreas urbana e rural do Brasil. Revista de

Economia e Sociologia Rural, Brasilia, v. 43, n. 1, p. 1-15, 2005. Disponível em:

<http://www.scielo.br/pdf/resr/v43n1/25834.pdf>. Acesso em: 17 jun. 2017.

https://doi.org/10.1590/S0103-20032005000100002

KUBOCA, K. Análise do tratamento das hepatites virais $B$ e $C$ nos usuários atendidos pelo

Sistema único de saúde no estado do Amapá. 37 f. 2010. Dissertação (Ciências Farmaceuticas) Universidade de São Paulo, Ribeirão Preto; 2010.

KUBOTA K, CAMPOS MSA, PEREIRA LRL. Análise da assistência à saúde aos pacientes com hepatites virais B e C no estado do Amapá. Revista de Ciência Farmacêutica Básica Aplicadas, São Paulo, v. 35, n. 4, p. 1-8, 2014. Disponível em: <http://serv-

bib.fcfar.unesp.br/seer/index.php/Cien_Farm/article/viewFile/3199/3199>. Acesso em: 16 mai. 2017.

MARGREITER, S. et al. Estudo de prevalência das hepatites virais b e c no município de PalhoçaSC. Revista de Saúde Pública, Rio de Janeiro, v. 8, n. 2, p. 1-11, 2015. Disponível em: <http://esp.saude.sc.gov.br/sistemas/revista/index.php/inicio/article/view/297/308>. Acesso em: 20 fev. 2017.

MORAIS, E. P.; RODRIGUES, R. A. P.; GERHARDT, T. E. Os idosos mais velhos no meio rural: realidade de vida e saúde de uma população do interior gaúcho. Texto contexto - enfermagem, Santa Catarina, v. 17, n. 2, p. 1-9, 2008. Disponível em: <http://www.scielo.br/pdf/tce/v17n2/21.pdf>. Acesso em: 17 jun. 2017. https://doi.org/10.1590/S0104-07072008000200021

OLIVEIRA, A. C. D. S.; FOCACCIA, R. Levantamento das hepatites B e C controle de infecção: procedimentos em instalações de manicure e pedicure em São Paulo, Brasil. Brazilian Journal of Infectious Diseases, Salvador, v. 14, n. 5, p. 502-507, 2010. https://doi.org/10.1016/S1413$\underline{\text { 8670(10)70100-8 }}$

OLIVEIRA, S. A. N. et al. A window of opportunity: declining rates of hepatitis B virus infection among injection drug users in Rio de Janeiro, and prospects for targeted 95 hepatitis B vaccination. Revista Panamericana de Salud Publica, São Paulo, v. 18, n. 4, p. 1-6, 2005. Disponível em:

<http://www.scielosp.org/pdf/rpsp/v18n4-5/28089.pdf>. Acesso em: 20 jun. 2017. https://doi.org/10.1590/S1020-49892005000900007

OMS. Organização Mundial da Saúde. Relatório global sobre hepatites de 2017. Brasília: OMS, 2017.

PALÁCIOS, V. R. C. et al. Leprosy and pregnancy in the State of Pará: an epidemiological perspective. Revista da Sociedade Brasileira de Medicina Tropical, Uberaba, v. 46, n. 4, p. 1-7, 2013. Disponível em: <http://www.scielo.br/pdf/rsbmt/v46n4/0037-8682-rsbmt-00-00-21.pdf >. Acesso em: 23 mar. 2017.

PAROLIN, M. B. et al. Prevalência de infecção pelo vírus da hepatite $C$ em pacientes com diabete melito tipo 2. Arquivo Gastroenterologia, São Paulo, v. 43, n. 2, p. 1-3, 2006. Disponível em:

<http://www.scielo.br/pdf/ag/v43n2/31125.pdf>. Acesso em: 10 abr. 2017. https://doi.org/10.1590/S0004-28032006000200003

PNUD. Programa das Nações Unidas para o Desenvolvimento. Atlas do Desenvolvimento Humano no Brasil. Brasília: PNUD, 2016. 
Análise espacial e epidemiológica de hepatites B e $\mathrm{C}$ e índice de desenvolvimento humano municipal, no estado do Pará
Nelson Veiga Gonçalves

Dayseanne Costa Vieira Claudia do Socorro Carvalho Miranda Vera Regina da Cunha Menezes Palácios Simone Beverly Nascimento da Costa Juan Andrade Guedes Bruno de Oliveira Santos Rodrigo Junior Farias da Costa Sheila Cristina Martins e Silva Rafael Aleixo Coelho de Oliveira

PUDELCO P, KOEHLER AE, BISETTO LHL. Impacto da vacinação na redução da hepatite B no Paraná. Revista Gaúcha de Enfermagem, Porto Alegre, v. 35, n. 1, p. 1-9, 2014. Disponível em: <http://www.scielo.br/pdf/rgenf/v35n1/pt_1983-1447-rgenf-35-01-00078.pdf>. Acesso em: 07 jun. 2017.

SESPA. Secretaria de Estado de Saúde Pública. Centros Regionais de Saúde. Belém: SESPA, 2016.

VIEIRA, G. D.; VENTURA, C. G.; SOUSA, C. M. Ocorrência e distribuição espacial da hepatite c em um estado da Amazônia Ocidental Brasileira. Arquivo Gastroenterologia, São Paulo, v. 51, n. 4, p.13. 2014. Disponível em: <http://www.scielo.br/pdf/ag/v51n4/0004-2803-ag-51-04-316.pdf. Acesso em: 13 mar. 2017.

TERRAULT, N. A. Sexual activity as a risk factor for hepatitis C. Hepatology, New York, v. 36, n. 5, p. 1-6, 2002. Disponível em: <https://www.ncbi.nlm.nih.gov/pubmed/12407582>. Acesso em: 12 mar. 2017. https://doi.org/10.1053/jhep.2002.36797

XIMENES, R. A. A. et al. Methodology of a nationwide cross-sectional survey of prevalence and epidemiological patterns of hepatitis A, B and C infection in Brazil. Caderno de Saúde Pública, São Paulo, v. 26, n. 9, p. 1-11, 2010. Disponível em: <http://www.scielo.br/pdf/csp/v26n9/03.pdf>. Acesso em: 16 mai. 2017.

WORLD BANK. The international bank for reconstruction and development. Washington: World bank, 2006. 